\title{
Genitourinary System Neoplasm
}

National Cancer Institute

\section{Source}

National Cancer Institute. Genitourinary System Neoplasm. NCI Thesaurus. Code C156482.

A benign or malignant neoplasm that affects the genitourinary system. 